\title{
Simulation of Sorbent-Enhanced Steam Methane Reforming and Limestone Calcination in Dual Turbulent Fluidized Bed Reactors - Supplementary Information
}

\author{
Arian Ebneyamini*, John R. Grace, Choon J. Lim, Naoko Ellis
}

Department of Chemical and Biological Engineering, University of British Columbia. Vancouver, Canada V6T 1 Z3

* Email: aebneyam@mail.ubc.ca

Table S.1. Governing equations for kinetic simulations

\begin{tabular}{|c|c|}
\hline $\begin{array}{c}\text { Dimensionless Form } \\
\text { of Gas Phase Mass } \\
\text { Balance }\end{array}$ & $\begin{array}{l}h=\frac{z}{L_{\text {Reactor }}} \text { and } P e=\frac{U L_{\text {Reactor }}}{D_{a}} \\
\frac{1}{P e} \frac{d^{2} F_{i}}{d h^{2}}-\frac{d F_{i}}{d h}+L_{\text {Reactor }}\left(\rho_{\text {Cat bed }}\left(\sum_{j=1}^{5} v_{i, j} r_{j}\right) A_{\text {Reactor }}+\pi N_{\text {Mem }} d_{\text {Mem }} J_{H_{2}, z}\right)+\frac{d F_{C O_{2}, D e s, z}}{d h}-\frac{d F_{C O_{2}, \text { Sor }, z}}{d h}=0 \\
\begin{array}{ll}\mathrm{BC} 1: F_{i, h=0}-F_{i, i n}=\left.\frac{1}{P e} \frac{d F_{i}}{d h}\right|_{h=0} \quad \text { BC2: }\left.\frac{d F_{i}}{d h}\right|_{h=L_{\text {Reator }}}=0 \quad \text { i: } \mathrm{CH}_{4}, \mathrm{CO}_{2}, \mathrm{CO}, \mathrm{H}_{2}, \mathrm{H}_{2} \mathrm{O}, \mathrm{O}_{2}, N_{2}\end{array}\end{array}$ \\
\hline $\begin{array}{l}\mathrm{CaCO}_{3} \text { Desorption } \\
\text { Mass Balance }\end{array}$ & 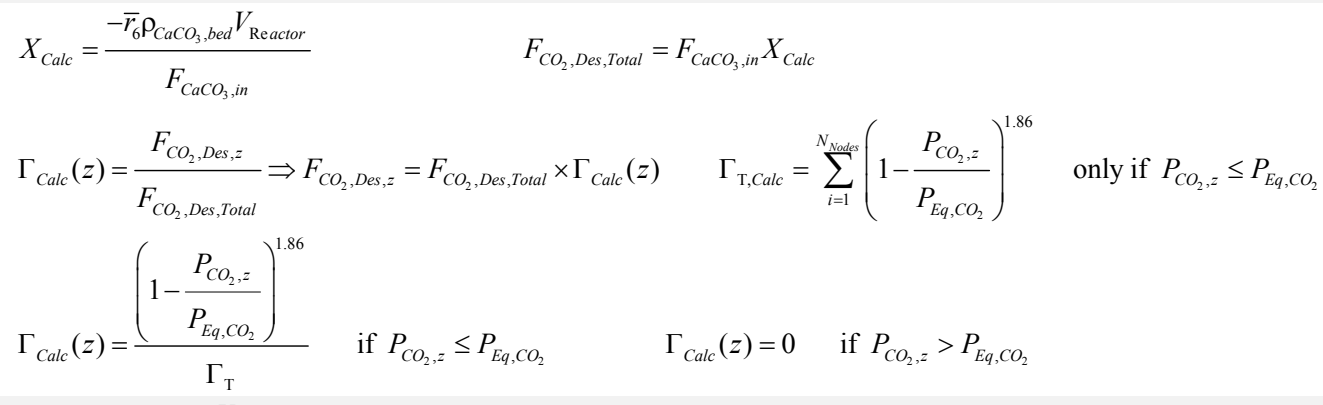 \\
\hline $\begin{array}{l}\text { CaO Sorption } \\
\text { Mass Balance }\end{array}$ & 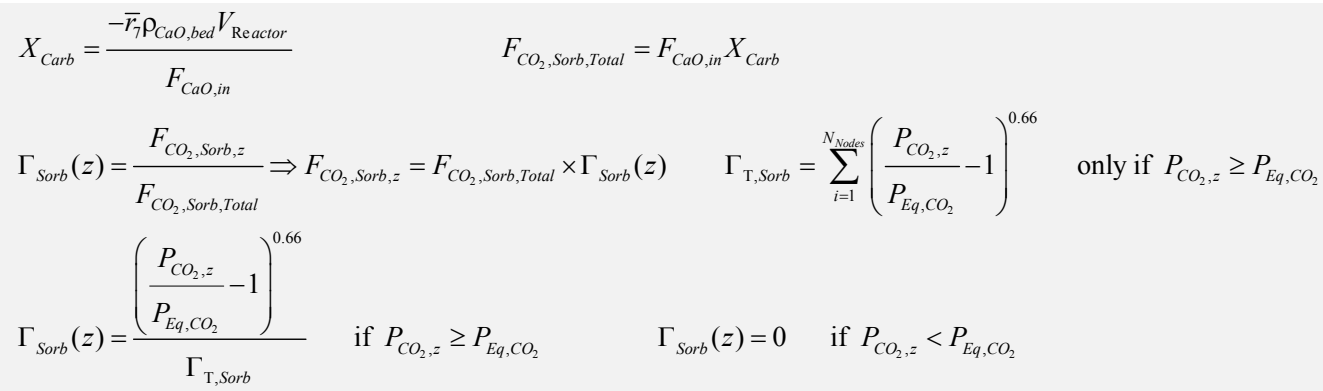 \\
\hline Pressure Drop & $\frac{d P_{t o t}}{d z}=\rho_{\text {solid }}\left(1-\varepsilon_{b e d}\right) g+\rho_{g a s} \varepsilon_{b e d} g$ \\
\hline Solid Concentrations & $\begin{array}{l}\rho_{\text {Cat,bed }}=\left(1-\varepsilon_{\text {bed }}\right) \rho_{\text {Solid }} \frac{m_{\text {Catalyst }}}{m_{\text {Solid }}} \\
\rho_{\text {CaCO }, \text { bed }}=\left(1-\varepsilon_{\text {bed }}\right) \rho_{\text {Solid }} \frac{m_{\text {CaCO }}}{m_{\text {Solid }}}\end{array}$ \\
\hline Bed Voidage ${ }^{1,2}$ & $\varepsilon_{\text {bed }}=\frac{U_{\text {Superficial }}+1}{U_{\text {Superficial }}+2}$ \\
\hline Permeation Flux & $J_{H_{2}, z}=\frac{\kappa_{H_{2}, P_{d}}}{\delta}\left(P_{H_{2}, \text { flim }, z}^{0.5}-P_{H_{2}, \text { Permeate }}^{0.5}\right)=\frac{k_{g, \text { Mem }}}{R T}\left(P_{H_{2}, \text { reformer }, z}-P_{H_{2}, \text { flilm }, z}\right)$ \\
\hline
\end{tabular}


Table S.2. Reactions considered for kinetics simulations

\begin{tabular}{|c|c|c|}
\hline Reaction & Stoichiometry & Rate Expression \\
\hline $\begin{array}{l}\text { Dry Methane Reforming } \\
(\mathrm{DMR})^{3,4}\end{array}$ & $\mathrm{CH}_{4}+\mathrm{CO}_{2} € 2 \mathrm{H}_{2}+2 \mathrm{CO}$ & $r_{1}=\frac{k_{1} K_{C_{4}, 1} K_{C_{2}, 1} P_{C H_{4}} P_{C_{2}}}{\left(1+K_{C_{4}, 1} P_{C H_{4}}+K_{C O_{2}, 1} P_{C O_{2}}\right)^{2}}\left(1-\frac{P_{C O}^{2} P_{H_{2}}^{2}}{K_{P, 1} P_{C H_{4}} P_{C O_{2}}}\right)$ \\
\hline $\begin{array}{l}\text { Reverse Water-Gas Shift } \\
\text { Reaction (RWGS) }\end{array}$ & $\mathrm{H}_{2}+\mathrm{CO}_{2} € \quad \mathrm{H}_{2} \mathrm{O}+\mathrm{CO}$ & $r_{2}=\frac{k_{2} K_{\mathrm{H}_{2}, 2} K_{\mathrm{CO}_{2}, 2} P_{\mathrm{H}_{2}} P_{\mathrm{CO}_{2}}}{\left(1+K_{\mathrm{H}_{2}, 2} P_{\mathrm{H}_{2}}+K_{\mathrm{CO}_{2}, 2} P_{\mathrm{CO}_{2}}\right)^{2}}\left(1-\frac{P_{\mathrm{CO}} P_{\mathrm{H}_{2} \mathrm{O}}}{K_{P_{2}} P_{\mathrm{H}_{2}} P_{\mathrm{CO}_{2}}}\right)$ \\
\hline $\begin{array}{l}\text { Steam Methane Reforming } \\
\text { (SMR) } 1^{5}\end{array}$ & $\mathrm{CH}_{4}+\mathrm{H}_{2} \mathrm{O} € 3 \mathrm{H}_{2}+\mathrm{CO}$ & $r_{3}=k_{3}\left(\frac{P_{C H_{4}} P_{H_{2} O}}{P_{H_{2}}^{2.5}}-\frac{P_{C O} P_{H_{2}}^{0.5}}{K_{3}}\right) / D E N^{2}$ \\
\hline SMR $2^{5}$ & $\mathrm{CH}_{4}+2 \mathrm{H}_{2} \mathrm{O} € 4 \mathrm{H}_{2}+\mathrm{CO}_{2}$ & $r_{4}=k_{4}\left(\frac{P_{\mathrm{CH}_{4}} P_{\mathrm{H}_{2} \mathrm{O}}^{2}}{P_{H_{2}}^{3.5}}-\frac{P_{\mathrm{CO}_{2}} P_{H_{2}}^{0.5}}{K_{3} K_{4}}\right) / D E N^{2}$ \\
\hline $\mathrm{CH}_{4}$ Combustion $^{6,7}$ & $\mathrm{CH}_{4}+2 \mathrm{O}_{2} \rightarrow 2 \mathrm{H}_{2} \mathrm{O}+\mathrm{CO}_{2}$ & $r_{5}=k_{5} P_{\mathrm{CH} 4} P_{\mathrm{O}_{2}}$ \\
\hline Limestone Calcination $^{8}$ & $\mathrm{CaCO}_{3} \rightarrow \mathrm{CaO}+\mathrm{CO}_{2}$ & $\begin{array}{l}\bar{r}_{6}=\frac{1}{M_{\mathrm{CaCO}_{3}}} \frac{d X}{d t} \\
\frac{d X_{\mathrm{Calc}}}{d t}=k_{6}\left(1-X_{\mathrm{Calc}}\right)^{2 / 3}\left(1-\frac{\bar{P}_{\mathrm{CO}_{2}}}{P_{E q, \mathrm{CO}_{2}}}\right)^{1.86}\end{array}$ \\
\hline \multirow[t]{2}{*}{ Lime Carbonation ${ }^{9}$} & \multirow[t]{2}{*}{$\mathrm{CaO}+\mathrm{CO}_{2} \rightarrow \mathrm{CaCO}_{3}$} & 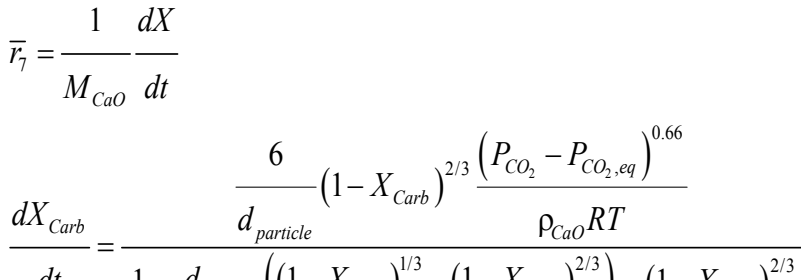 \\
\hline & & $d t \quad \frac{1}{k_{7}}+\frac{d_{\text {particle }}\left(\left(1-X_{\text {Carb }}\right)^{1 / 3}-\left(1-X_{\text {Carb }}\right)^{2 / 3}\right)}{2 D_{\text {eff }}}+\frac{\left(1-X_{\text {Carb }}\right)^{2 / 3}}{k_{g, \text { Particle }}}$ \\
\hline
\end{tabular}


Table S.3. Rate parameters for reactions considered for kinetics simulations

\begin{tabular}{|c|c|c|}
\hline Reaction & \multicolumn{2}{|c|}{ Reaction Rate Parameters } \\
\hline $\mathrm{DMR}^{3,4}$ & $\begin{array}{l}k_{1}\left[\frac{\mathrm{mol}}{\mathrm{kg}_{\mathrm{cat}} \cdot \mathrm{s}}\right]=1.29 \times 10^{6} \operatorname{Exp}\left(\frac{-102065}{R T}\right) \\
K_{\mathrm{CH}_{4}, 1}\left[\mathrm{bar}^{-1}\right]=2.566 \times 10^{-2} \operatorname{Exp}\left(\frac{40684}{R T}\right)\end{array}$ & $\begin{array}{l}K_{\mathrm{CO}_{2}, 1}\left[\mathrm{bar}^{-1}\right]=2.576 \times 10^{-2} \operatorname{Exp}\left(\frac{37641}{R T}\right. \\
K_{P, 1}\left[\mathrm{bar}^{2}\right]=6.96 \times 10^{14} \operatorname{Exp}\left(\frac{-259660}{R T}\right)\end{array}$ \\
\hline $\mathrm{RWGS}^{3,4}$ & $\begin{array}{l}k_{2}\left[\frac{\mathrm{mol}}{\mathrm{kg}_{\text {cat }} . \mathrm{s}}\right]=0.35 \times 10^{6} \operatorname{Exp}\left(\frac{-81030}{R T}\right) \\
K_{\mathrm{H}_{2}, 2}\left[\mathrm{bar}^{-1}\right]=1.474 \operatorname{Exp}\left(\frac{6025}{R T}\right)\end{array}$ & $\begin{array}{l}K_{\mathrm{CO}_{2}, 2}\left[\mathrm{bar}^{-1}\right]=0.57 \operatorname{Exp}\left(\frac{9262}{R T}\right) \\
K_{P, 2}[-]=56.497 \operatorname{Exp}\left(\frac{-36580}{R T}\right)\end{array}$ \\
\hline SMR $1^{5}$ & $\begin{array}{l}\left.D E N=1+K_{C O, S M R} P_{C O}+K_{H_{2}, S M R} P_{H_{2}}+K_{\mathrm{CH}_{4, S H R}} P_{C_{4}}+\frac{K_{H_{2}}}{R T}\right) \\
k_{3}\left[\frac{k m o l \cdot b a r^{0.5}}{k g_{c a t} \cdot s}\right]=1.174 \times 10^{12} \operatorname{Exp}\left(\frac{-240100}{R T}\right) \\
K_{3}\left[\text { bar }^{2}\right]=\operatorname{Exp}\left[\left(\frac{-26830}{T}\right)+30.11\right]\end{array}$ & 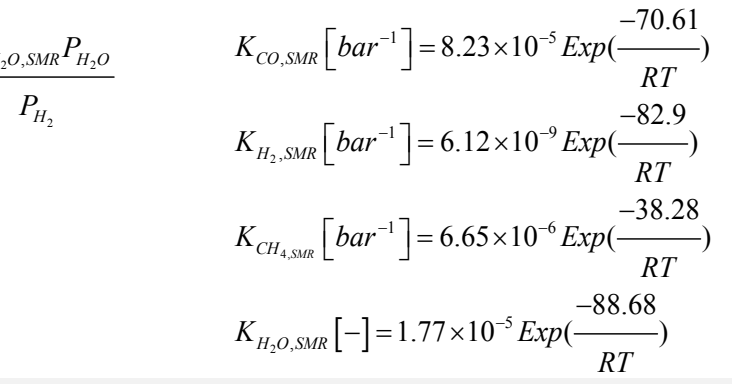 \\
\hline SMR $2^{5}$ & $k_{4}\left[\frac{k m o l . b a r^{0.5}}{k g_{\text {cat }} . s}\right]=2.83 \times 10^{11} \operatorname{Exp}\left(\frac{-24390}{R T}\right.$ & $-K_{4}[-]=\operatorname{Exp}\left[\left(\frac{4400}{T}\right)-4.036\right]$ \\
\hline $\begin{array}{c}\text { Methane } \\
\text { Combustion }^{6,7}\end{array}$ & $k_{5}\left[\frac{m o l}{k g_{c a t} \cdot s \cdot b a r^{2}}\right]$ & $=1.1 \times 10^{13} \exp \left(\frac{-166000}{R T}\right)$ \\
\hline $\begin{array}{l}\text { Limestone } \\
\text { Calcination }^{8}\end{array}$ & $k_{6}\left[s^{-1}\right]=5.6$ & $1 \times 10^{5} \operatorname{Exp}\left(\frac{-150000}{R T}\right)$ \\
\hline Lime Carbonation ${ }^{9}$ & $\begin{array}{c}k_{7}\left[\frac{m}{s}\right]=3.05 \operatorname{Exp}\left(\frac{-32600}{R T}\right) \\
\tau[-]=5 \\
\varepsilon_{\text {Pore }}[-]=0.5\end{array}$ & $\begin{array}{l}D_{P L}\left[\frac{m^{2}}{s}\right]=7.7 \times 10^{-9} \\
r_{\text {Pore }}[m]=10 \times 10^{-9}\end{array}$ \\
\hline $\mathrm{H}_{2}$ Permeation $^{10}$ & $\kappa_{H_{2}, P d}\left[\frac{m o l}{m . s . b a r^{0.5}}\right]$ & $=3.43 \times 10^{-5} \times \exp \left(\frac{-9180}{R T}\right)$ \\
\hline
\end{tabular}


Table S.4. Correlations and supporting equation applied for kinetics simulations

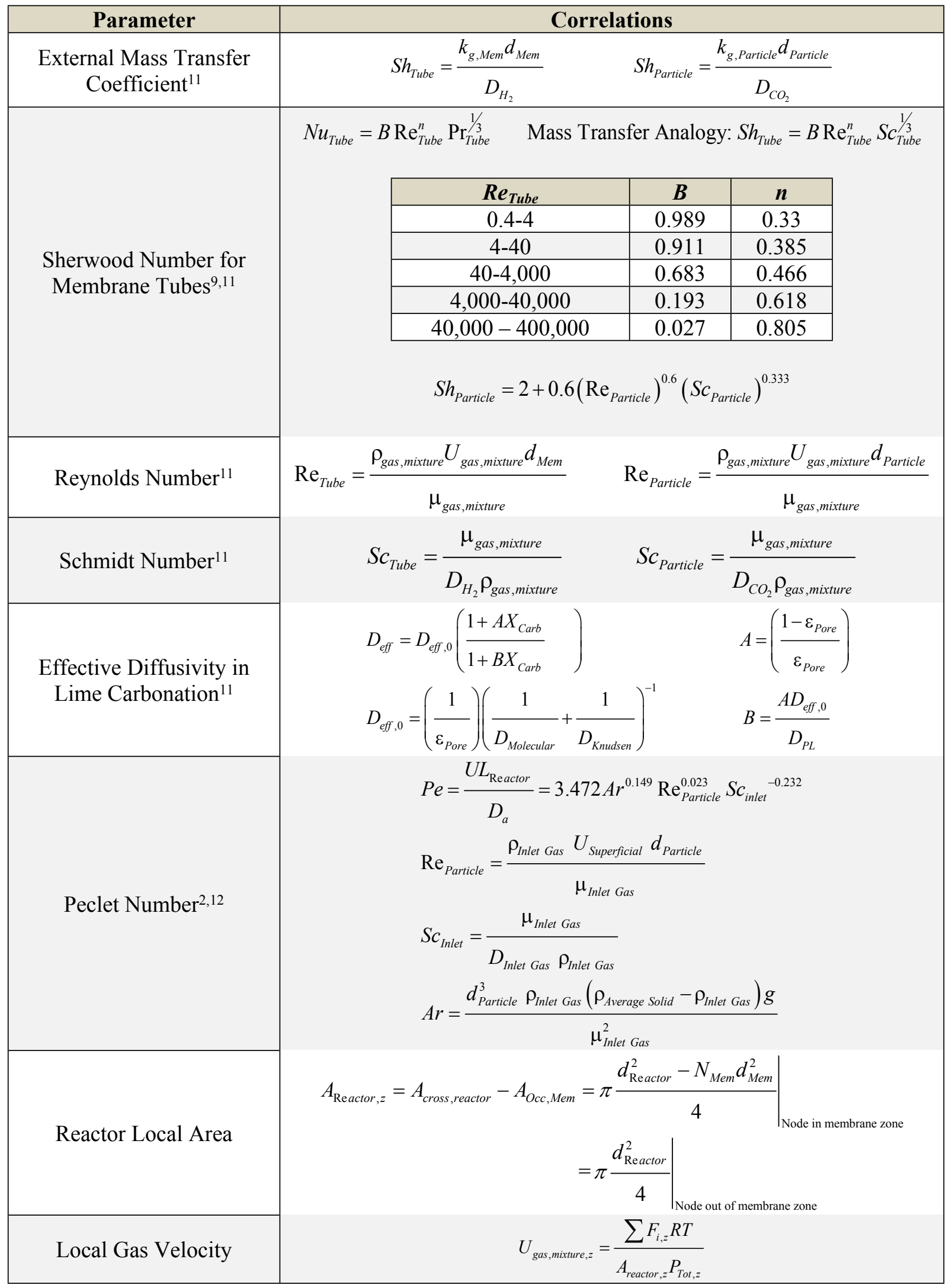


Table S.5. Summary of ASPEN plus and kinetic simulation results for reformers

\begin{tabular}{|c|c|c|c|c|c|c|c|c|}
\hline \multicolumn{9}{|c|}{ Summary of Operating Conditions } \\
\hline & \multicolumn{4}{|c|}{ ASPEN Simulation } & \multicolumn{4}{|c|}{ Kinetic Simulation } \\
\hline Reactor Temperature $\left({ }^{\circ} \mathrm{C}\right)$ & \multicolumn{4}{|c|}{650} & \multicolumn{4}{|c|}{650} \\
\hline Reactor Pressure (bars) & \multicolumn{4}{|c|}{3} & \multicolumn{4}{|c|}{3} \\
\hline Steam/Methane Molar Feed Ratio & \multicolumn{4}{|c|}{3.25} & \multicolumn{4}{|c|}{3.25} \\
\hline $\mathrm{CaO}$ Inlet Flow Rate $\left(\mathrm{mol} . \mathrm{s}^{-1}\right)^{*}$ & \multicolumn{4}{|c|}{0.041} & \multicolumn{4}{|c|}{0.21} \\
\hline \multicolumn{9}{|c|}{ Steam Methane Reformer } \\
\hline & \multicolumn{4}{|c|}{ ASPEN Simulation } & \multicolumn{4}{|c|}{ Kinetic Simulation } \\
\hline Off-gas Flow Rate (mol.s $\left.{ }^{-1}\right)$ & \multicolumn{4}{|c|}{0.271} & \multicolumn{4}{|c|}{0.270} \\
\hline \multirow{3}{*}{ Off-gas Molar Composition } & $\mathrm{H}_{2}$ & $47.41 \%$ & $\mathrm{CO}$ & $5.19 \%$ & $\mathrm{H}_{2}$ & $46.63 \%$ & $\mathrm{CO}$ & $5.01 \%$ \\
\hline & $\mathrm{CO}_{2}$ & $7.96 \%$ & $\mathrm{CH}_{4}$ & $4.19 \%$ & $\mathrm{CO}_{2}$ & $7.9 \%$ & $\mathrm{CH}_{4}$ & $4.55 \%$ \\
\hline & $\mathrm{H}_{2} \mathrm{O}$ & $35.25 \%$ & $\mathrm{~N}_{2}$ & $0 \%$ & $\mathrm{H}_{2} \mathrm{O}$ & $35.91 \%$ & $\mathrm{~N}_{2}$ & $0 \%$ \\
\hline Reactor Heat Duty (kW) & \multicolumn{8}{|c|}{7.049} \\
\hline Fuel Combustion Energy $\left(\mathrm{kJ} \cdot \mathrm{mol}^{-1}\right)^{* *}$ & \multicolumn{8}{|c|}{727.64} \\
\hline Fuel Requirement (mol.s $\left.\mathrm{s}^{-1}\right)$ & \multicolumn{8}{|c|}{$9.69 \times 10^{-3}$} \\
\hline \multicolumn{9}{|c|}{ Sorbent-Enhanced Steam Methane Reformer } \\
\hline & \multicolumn{4}{|c|}{ ASPEN Simulation } & \multicolumn{4}{|c|}{ Kinetic Simulation } \\
\hline Off-gas Flow Rate (mol.s $\left.{ }^{-1}\right)$ & \multicolumn{4}{|c|}{0.245} & \multicolumn{4}{|c|}{0.245} \\
\hline \multirow{3}{*}{ Off-gas Molar Composition } & $\mathrm{H}_{2}$ & $69.96 \%$ & $\mathrm{CO}$ & $0.46 \%$ & $\mathrm{H}_{2}$ & $67.84 \%$ & $\mathrm{CO}$ & $0.61 \%$ \\
\hline & $\mathrm{CO}_{2}$ & $0.36 \%$ & $\mathrm{CH}_{4}$ & $1.59 \%$ & $\mathrm{CO}_{2}$ & $0.53 \%$ & $\mathrm{CH}_{4}$ & $2.12 \%$ \\
\hline & $\mathrm{H}_{2} \mathrm{O}$ & $27.63 \%$ & $\mathrm{~N}_{2}$ & $0 \%$ & $\mathrm{H}_{2} \mathrm{O}$ & $28.9 \%$ & $\mathrm{~N}_{2}$ & $0 \%$ \\
\hline Reactor Heat Duty $(\mathrm{kW})$ & \multicolumn{8}{|c|}{1.12} \\
\hline Fuel Combustion Energy (kJ.mol-1) & \multicolumn{8}{|c|}{727.64} \\
\hline Fuel Requirement $\left(\mathrm{mol}_{\mathrm{s}} \mathrm{s}^{-1}\right)$ & \multicolumn{8}{|c|}{$1.54 \times 10^{-3}$} \\
\hline
\end{tabular}

* $\mathrm{CaO}$ feed flow in ASPEN simulation was set to be equal to the carbonated lime predicted by the kinetic simulation (SE-SMR)

** Calculated using RGibbs (with stoichiometric $\mathrm{CH}_{4}$ and $\mathrm{O}_{2}$ feedstock at $25^{\circ} \mathrm{C}$ ), operating at reformer temperature and pressure. 
Table S.6. Summary of ASPEN plus and kinetic simulation results for calciners

\begin{tabular}{|c|c|c|c|c|c|c|c|c|}
\hline \multicolumn{9}{|c|}{ Summary of Operating Conditions } \\
\hline & \multicolumn{4}{|c|}{ ASPEN Simulation } & \multicolumn{4}{|c|}{ Kinetic Simulation } \\
\hline MRC-CAL Temperature $\left({ }^{\circ} \mathrm{C}\right)$ & \multicolumn{4}{|c|}{825} & \multicolumn{4}{|c|}{825} \\
\hline Steam Calciner Temperature $\left({ }^{\circ} \mathrm{C}\right)$ & \multicolumn{4}{|c|}{880} & \multicolumn{4}{|c|}{880} \\
\hline Reactor Pressure (bars) & \multicolumn{4}{|c|}{3} & \multicolumn{4}{|c|}{3} \\
\hline Gas Feed Ratio (mol.s $\left.{ }^{-1}\right)$ & \multicolumn{4}{|c|}{0.137} & \multicolumn{4}{|c|}{0.137} \\
\hline MRC-CAL Gas Feed Composition & \multicolumn{4}{|c|}{$60.5 \% \mathrm{CH}_{4}+37.5 \% \mathrm{O}_{2}+2 \% \mathrm{~N}_{2}$} & \multicolumn{4}{|c|}{$60.5 \% \mathrm{CH}_{4}+37.5 \% \mathrm{O}_{2}+2 \% \mathrm{~N}_{2}$} \\
\hline Steam Calciner Gas Feed Composition & \multicolumn{4}{|c|}{$100 \%$ Steam } & \multicolumn{4}{|c|}{$100 \%$ Steam } \\
\hline $\mathrm{CaCO}_{3}$ Inlet Flow Rate $\left(\mathrm{mol} \mathrm{s}^{-1}\right)$ & \multicolumn{4}{|c|}{0.041} & \multicolumn{4}{|c|}{0.041} \\
\hline \multicolumn{9}{|c|}{ MRC-CAL Calciner } \\
\hline & \multicolumn{4}{|c|}{ ASPEN Simulation } & \multicolumn{4}{|c|}{ Kinetic Simulation } \\
\hline Off-gas Flow Rate (mol.s $\left.\mathrm{s}^{-1}\right)$ & \multicolumn{4}{|c|}{0.289} & \multicolumn{4}{|c|}{0.289} \\
\hline \multirow{3}{*}{ Off-gas Molar Composition } & $\mathrm{H}_{2}$ & $43.67 \%$ & $\mathrm{CO}$ & $32.94 \%$ & $\mathrm{H}_{2}$ & $43.7 \%$ & $\mathrm{CO}$ & $33.1 \%$ \\
\hline & $\mathrm{CO}_{2}$ & $9.35 \%$ & $\mathrm{CH}_{4}$ & $0.66 \%$ & $\mathrm{CO}_{2}$ & $9.1 \%$ & $\mathrm{CH}_{4}$ & $0.6 \%$ \\
\hline & $\mathrm{H}_{2} \mathrm{O}$ & $12.44 \%$ & $\mathrm{~N}_{2}$ & $0.94 \%$ & $\mathrm{H}_{2} \mathrm{O}$ & $12.6 \%$ & $\mathrm{~N}_{2}$ & $0.9 \%$ \\
\hline Reactor Heat Duty $(\mathrm{kW})$ & \multicolumn{8}{|c|}{0.325} \\
\hline Fuel Combustion Energy $\left(\mathrm{kJ} . \mathrm{mol}^{-1}\right)^{*}$ & \multicolumn{8}{|c|}{703.62} \\
\hline Fuel Requirement (mol.s $\left.\mathrm{s}^{-1}\right)$ & \multicolumn{8}{|c|}{$0.461 \times 10^{-3}$} \\
\hline \multicolumn{9}{|c|}{ Steam Calciner } \\
\hline & \multicolumn{4}{|c|}{ ASPEN Simulation } & \multicolumn{4}{|c|}{ Kinetic Simulation } \\
\hline Off-gas Flow Rate $\left(\mathrm{mol} . \mathrm{s}^{-1}\right)$ & \multicolumn{4}{|c|}{0.178} & \multicolumn{4}{|c|}{0.178} \\
\hline & $\mathrm{H}_{2}$ & $0 \%$ & $\mathrm{CO}$ & $0 \%$ & $\mathrm{H}_{2}$ & $0 \%$ & $\mathrm{CO}$ & $0 \%$ \\
\hline Off-gas Molar Composition & $\mathrm{CO}_{2}$ & $23.08 \%$ & $\mathrm{CH}_{4}$ & $0 \%$ & $\mathrm{CO}_{2}$ & $23.01 \%$ & $\mathrm{CH}_{4}$ & $0 \%$ \\
\hline & $\mathrm{H}_{2} \mathrm{O}$ & $76.92 \%$ & $\mathrm{~N}_{2}$ & $0 \%$ & $\mathrm{H}_{2} \mathrm{O}$ & $76.99 \%$ & $\mathrm{~N}_{2}$ & $0 \%$ \\
\hline Reactor Heat Duty $(\mathrm{kW})$ & & & & & & & & \\
\hline Fuel Combustion Energy $\left(\mathrm{kJ} \cdot \mathrm{mol}^{-1}\right)$ & & & & & & & & \\
\hline Fuel Requirement (mol.s $\left.{ }^{-1}\right)$ & & & & 1.02 & $10^{-2}$ & & & \\
\hline
\end{tabular}

* Calculated using RGibbs (with stoichiometric $\mathrm{CH}_{4}$ and $\mathrm{O}_{2}$ feedstock at $25^{\circ} \mathrm{C}$ ), operating at calciner temperature and pressure. 


\section{Nomenclature}

\begin{tabular}{|c|c|}
\hline$A_{\text {Reactor }}$ & Reactor cross-sectional area $\left(m^{2}\right)$ \\
\hline$A_{A C C, \mathrm{Mem}}$ & Area occupied by membrane tubes $\left(m^{2}\right)$ \\
\hline$A r$ & Archimedes number $=d_{\text {Particle }}^{3} \cdot \rho_{\text {Inlet-Gas }} \cdot\left(\rho_{\text {Average-Solid }}-\rho_{\text {Inlet-Gas }}\right) \cdot g \cdot \mu_{\text {Inlet-Gas }}^{-2}$ \\
\hline$C_{p}$ & Specific Heat $\left(J \cdot \mathrm{kg}^{-1} \cdot K^{-1}\right)$ \\
\hline$D_{a}$ & Axial dispersion coefficient of gas $\left(\mathrm{m}^{2} \cdot \mathrm{s}^{-1}\right)$ \\
\hline$D_{H_{2}}$ & Molecular diffusivity of $\mathrm{H}_{2}\left(m^{2} \cdot s^{-1}\right)$ \\
\hline$D_{\mathrm{CO}_{2}}$ & Molecular diffusivity of $\mathrm{CO}_{2}\left(m^{2} \cdot s^{-I}\right)$ \\
\hline$D_{e f f}$ & Effective $\mathrm{CO}_{2}$ diffusivity in sorbent $\left(\mathrm{m}^{2} \cdot \mathrm{s}^{-1}\right)$ \\
\hline$D_{\text {Molecular }}$ & Molecular diffusion coefficient of $\mathrm{CO}_{2}$ in sorbent $\left(\mathrm{m}^{2} \cdot \mathrm{s}^{-1}\right)$ \\
\hline$D_{\text {Knudsen }}$ & $\mathrm{CO}_{2}$ diffusivity in product layer $\left(\mathrm{m}^{2} \cdot \mathrm{s}^{-1}\right)$ \\
\hline$D_{P L}$ & Molecular diffusion coefficient of $\mathrm{CO}_{2}$ in sorbent $\left(\mathrm{m}^{2} \cdot \mathrm{s}^{-1}\right)$ \\
\hline$d_{\text {Particle }}$ & Particle diameter $(m)$ \\
\hline$d_{M e m}$ & Membrane Tube diameter $(m)$ \\
\hline$F_{i}$ & Molar flow rate of component $i\left(\right.$ mol.s $\left.\mathrm{s}^{-I}\right)$ \\
\hline$F_{\mathrm{CO}_{2}, \mathrm{Des}, \mathrm{z}}$ & Local flow rate of $\mathrm{CO}_{2}$ released by calcination $\left(\right.$ mol. $\left.^{-1}\right)$ \\
\hline$F_{\mathrm{CO}_{2}, \mathrm{Sorb}, z}$ & Local flow rate of $\mathrm{CO}_{2}$ removed by carbonation $\left(\mathrm{mol} . \mathrm{s}^{-1}\right)$ \\
\hline$g$ & Acceleration of gravity $\left(m \cdot s^{-2}\right)$ \\
\hline$h$ & Dimensionless reactor length coordinate $\left(=z \cdot L_{\text {Reactor }}^{-1}\right)(-)$ \\
\hline$h_{\text {Tube }}$ & Convective heat transfer coefficient $\left(W \cdot m^{-2} \cdot K^{-1}\right)$ \\
\hline$J_{H_{2}, z}$ & Hydrogen Permeation Flux in Membranes $\left(\operatorname{mol} . m^{-2} \cdot s^{-1}\right)$ \\
\hline$k_{j}$ & Rate constant of reactions $j: 1-7$ (units vary) \\
\hline$k_{h, \text { Tube }}$ & Thermal conductivity of membrane tubes $\left(W \cdot m^{-1} \cdot K^{-1}\right)$ \\
\hline$K_{j}$ & Equilibrium constant for reaction $j\left(b a r^{2}\right)$ \\
\hline
\end{tabular}




\begin{tabular}{|c|c|}
\hline$K_{i, j}$ & Adsorption constant for component $i$ in reaction $j$ (unit varies) \\
\hline$K_{p, j}$ & Equilibrium constants for reaction $j: 1-5$ (units vary) \\
\hline$K_{i, S M R}$ & Adsorption constant for component $i$ in SMR reactions, $i$ (units vary) \\
\hline$k_{g, M e m}$ & External mass transfer coefficient for membranes $\left(m \cdot s^{-1}\right)$ \\
\hline$k_{g, \text { Particle }}$ & External mass transfer coefficient for particles $\left(m \cdot s^{-1}\right)$ \\
\hline$L_{\text {Reactor }}$ & Reactor length $(m)$ \\
\hline$M_{\mathrm{CaCO}_{3}}$ & $\mathrm{CaCO}_{3}$ molecular weight $\left(\mathrm{kg} \cdot \mathrm{mol}^{-1}\right)$ \\
\hline$M_{\mathrm{CaO}}$ & $\mathrm{CaO}$ molecular weight (kg.mol $\left.{ }^{-1}\right)$ \\
\hline$N_{M e m}$ & Number of membrane tubes (-) \\
\hline$N u_{\text {Tube }}$ & Nusselt number $=h_{\text {Tube }} \cdot L_{\text {Tube }} \cdot k_{h, \text { Tube }}^{-1}(-)$ \\
\hline $\bar{P}_{\mathrm{CO}_{2}}$ & Average $\mathrm{CO}_{2}$ partial pressure in reactor (bars) \\
\hline$P_{i}$ & Partial pressure of component $i$ (bars) \\
\hline$P_{H_{2}, \text { reformer }, z}$ & Local partial pressure of hydrogen in reformer (bars) \\
\hline$P_{H_{2}, f i l m, z}$ & Local partial pressure of hydrogen in membrane film (bars) \\
\hline$P_{t o t}$ & Total pressure of component $i$ (bars) \\
\hline$P e$ & Peclet number $=U \cdot L_{\text {Reactor }} \cdot D_{a}^{-1}(-)$ \\
\hline$P_{E q, C O_{2}}$ & Partial pressure of $\mathrm{CO}_{2}$ in equilibrium with lime/limestone (bars) \\
\hline $\operatorname{Pr}_{\text {Tube }}$ & Prandtl number for single membrane tube $=\mu \cdot C_{p} \cdot k_{h, \text { Tube }}^{-1}(-)$ \\
\hline$R$ & Gas constant $\left(J . m o l^{-1} \cdot K^{-1}\right)$ \\
\hline $\mathrm{Re}_{\text {Particle }}$ & Reynolds number for single particle $=\rho_{\text {Inlet-Gas }} \cdot U_{\text {Superficial }} \cdot d_{\text {Particle }} \cdot \mu_{\text {Inlet-Gas }}^{-1}$ \\
\hline $\operatorname{Re}_{\text {Tube }}$ & Reynolds number for single tube $=\rho_{\text {Inlet-Gas }} \cdot U_{\text {Superficial }} \cdot d_{\text {Membrane }} \cdot \mu_{\text {Inlet-Gas }}^{-1}$ \\
\hline$r_{j}$ & Reaction rates $j: 1-5\left(\mathrm{~mol}_{\mathrm{kg} \mathrm{cat}}{ }^{-1} \cdot \mathrm{s}^{-1}\right)$ and 6-7 $\left(\mathrm{mol} . \mathrm{kg} \mathrm{sorb}{ }^{-1} . \mathrm{s}^{-1}\right)$ \\
\hline$r_{\text {pore }}$ & Mean sorbent pore diameter $(m)$ \\
\hline$S h_{\text {Tube }}$ & Sherwood number for single membrane tube $=k_{g, \mathrm{Mem}} d_{\mathrm{Mem}} \cdot D_{\mathrm{H}_{2}}^{-1}(-)$ \\
\hline$S h_{\text {Particle }}$ & Sherwood number for single membrane tube $=k_{g, \text { Particle }} d_{\text {Particle }} \cdot D_{\mathrm{CO}_{2}}^{-1}(-)$ \\
\hline
\end{tabular}




$\begin{array}{ll}S c_{\text {inlet }} & \text { Schmidt number at reactor inlet }=\mu_{\text {Inlet }- \text { Gas }} \cdot D_{\text {Molecular }- \text { Inlet Gas }}^{-1} \cdot \rho_{\text {Inlet Gas }}^{-1}(-) \\ S c_{\text {Tube }} & \text { Schmidt number for tubes }=\mu_{\text {gas }, \text { mixture }} \cdot D_{H_{2}}^{-1} \cdot \rho_{\text {gas, mixture }}^{-1}(-) \\ S c_{\text {inlet }} & \text { Schmidt number for particles }=\mu_{\text {gas,mixture }} \cdot D_{\mathrm{CO}_{2}}^{-1} \cdot \rho_{\text {gas, mixture }}^{-1}(-) \\ T & \text { Operating temperature }\left(\mathrm{K} \text { or }{ }^{\circ} \mathrm{C}\right) \\ t & \text { Time }(s) \\ U_{\text {Superficial }} & \text { Superficial gas velocity }\left(m \cdot s^{-1}\right) \\ v_{i, j} & \text { Stoichiometric coefficient of component } i \text { in reaction } j \\ X_{\text {Calc }} & \text { Mean sorbent calcination extent }(-) \\ X_{\text {Carb }} & \text { Mean sorbent carbonation extent }(-) \\ z & \text { Reactor length coordinate }(m)\end{array}$

\section{Greek Letters}

\begin{tabular}{|c|c|}
\hline$\varepsilon_{b e d}$ & Bed voidage (-) \\
\hline$\varepsilon_{\text {Pore }}$ & Sorbent Porosity (-) \\
\hline$\kappa_{H_{2}, P d}$ & Hydrogen Permeability in Palladium-Based Membranes $\left(\right.$ mol. $\left.^{-1} . s^{-1} \cdot b a r^{-0.5}\right)$ \\
\hline$\mu$ & Gas viscosity $\left(k g \cdot m^{-1} \cdot s^{-1}\right)$ \\
\hline$\rho$ & Density $\left(\mathrm{kg} \cdot \mathrm{m}^{-3}\right)$ \\
\hline$\rho_{\mathrm{CaO}}$ & $\mathrm{CaO}$ molar density $\left(\right.$ mol. $\left.m^{-3}\right)$ \\
\hline$\rho_{\text {Cat }, b e d}$ & Catalyst bed concentration $\left(\mathrm{kg} \cdot \mathrm{m}^{-3}\right)$ \\
\hline$\rho_{\text {CaO,bed }}$ & $\mathrm{CaO}$ bed concentration $\left(\mathrm{kg} \cdot \mathrm{m}^{-3}\right)$ \\
\hline$\rho_{\mathrm{CaCO}_{3}, \text { bed }}$ & $\mathrm{CaCO}_{3}$ bed concentration $\left(\mathrm{kg} \cdot \mathrm{m}^{-3}\right)$ \\
\hline$\rho_{\text {Solid }}$ & Average solid $\left(\mathrm{CaO}, \mathrm{CaCO}_{3}\right.$, Catalyst $)$ density $\left(\mathrm{kg} \cdot \mathrm{m}^{-3}\right)$ \\
\hline$\delta$ & Membrane Active Layer Thickness $(m)$ \\
\hline$\tau$ & Sorbent tortuosity factor (-) \\
\hline$\Gamma_{\text {Calc }}(z)$ & Desorption distribution factor (-) \\
\hline
\end{tabular}


$\Gamma_{\text {Carb }}(z) \quad$ Desorption distribution factor (-)

\section{Subscripts}

$\begin{array}{ll}\text { Calc } & \text { Calcination } \\ \text { Carb } & \text { Carbonation } \\ \text { Cat } & \text { Catalyst } \\ \text { in } & \text { Gas/Solid Inlet } \\ 0 & \text { Value at reactor entrance }(\mathrm{z}=0)\end{array}$

\section{References}

(1) King, D. F. Estimation of Dense Bed Voidage in Fast and Slow Fluidized Beds of FCC Catalyst. In Fluidization VI (eds. J.R. Grace, L.W. Schemilt, \& M.A. Bergougnou); New York: Engineering Foundation, 1989; pp 1-8.

(2) Abba, I. A. A Generalized Fluidized Bed Reactor Model across the Flow Regimes, University of British Columbia, Ph.D. Thesis, 2001.

(3) Paripatyadar, S. A.; Richardson, J. T. Carbon Dioxide Reforming of Methane with Supported Rhodium. Appl. Catal. 1990, 61, 293-309.

(4) Benguerba, Y.; Dehimi, L.; Virginie, M.; Dumas, C.; Ernst, B. Numerical Investigation of the Optimal Operative Conditions for the Dry Reforming Reaction in a Fixed-Bed Reactor: Role of the Carbon Deposition and Gasification Reactions. React. Kinet. Mech. Catal. 2015, 115 (2), 483497. https://doi.org/10.1007/s11144-015-0849-9.

(5) Xu, J. G.; Froment, G. F. Methane Steam Reforming, Methanation and Water-Gas Shift . I. Intrinsic Kinetics. AIChE J. 1989, 35 (1), 88-96. https://doi.org/10.1002/aic.690350109.

(6) Chen, Z.; Po, F.; Grace, J. R.; Jim Lim, C.; Elnashaie, S. S. E. H.; Mahecha-Botero, A.; Rakib, M.; Shirasaki, Y.; Yasuda, I. Sorbent-Enhanced/Membrane-Assisted Steam-Methane Reforming. Chem. Eng. Sci. 2008, 63 (1), 170-182. https://doi.org/10.1016/j.ces.2007.09.031. 
(7) Jin, W.; Gu, X.; Li, S.; Huang, P.; Xu, N.; Shi, J. Experimental and Simulation Study on a Catalyst Packed Tubular Dense Membrane Reactor for Partial Oxidation of Methane to Syngas. Chem. Eng. Sci. 2000, 55 (14), 2617-2625. https://doi.org/10.1016/S0009-2509(99)00542-4.

(8) Li, Z. S.; Cai, N. S. Modeling of Multiple Cycles for Sorption-Enhanced Steam Methane Reforming and Sorbent Regeneration in Fixed Bed Reactor. Energy and Fuels 2007, 21 (5), 29092918. https://doi.org/10.1021/ef070112c.

(9) Johnsen, K. Sorption-Enhanced Steam Methane Reforming in Fluidized Bed Reactors, Norwegian University of Science and Technology, Ph.D. Thesis, 2006.

(10) Chen, Y.; Mahecha-Botero, A.; Lim, C. J.; Grace, J. R.; Zhang, J.; Zhao, Y.; Zheng, C. Hydrogen Production in a Sorption-Enhanced Fluidized-Bed Membrane Reactor: Operating Parameter Investigation. Ind. Eng. Chem. Res. 2014, 53 (14), 6230-6242. https://doi.org/10.1021/ie500294k.

(11) R. Welty, J.; E. Wicks, C.; E. Wilson, R.; Rorrer, G. Fundamentals of Momentum, Heat and Mass Transfer; 1970; Vol. 13. https://doi.org/10.1016/0017-9310(70)90063-3.

(12) Thompson, M. L.; Bi, H.; Grace, J. R. A Generalized Bubbling/Turbulent Fluidized-Bed Reactor Model. Chem. Eng. Sci. 1999, 54 (13-14), 2175-2185. 